\title{
A novel approach to fuzzy soft sets in decision making based on grey relational analysis and MYCIN certainty factor
}

\author{
Ningxin Xie ${ }^{1}$ Yu Han ${ }^{2}$ Zhaowen $\mathrm{Li}^{2}{ }^{*}$ \\ ${ }^{1}$ College of Information Science and Engineering, Guangxi University for Nationalities, Nanning, Guangxi \\ 530006, P.R.China \\ E-mail:ningxinxie100@126.com \\ ${ }^{2}$ College of Science, Guangxi University for Nationalities, Nanning, Guangxi 530006, P.R.China \\ E-mail:yuhan0124@126.com,lizhaowen8846@126.com
}

Received 9 January 2015

Accepted 7 August 2015

\begin{abstract}
This paper proposes a novel approach to decision making based on grey relational analysis and MYCIN certainty factor, which describes decision making problems with fuzzy soft sets. Firstly, we utilize grey relational analysis to obtain the grey mean relational degree, and the uncertain degree of various parameters is acquired. On the basis of uncertain degree, we obtain the essence uncertainty factor of each independent alternative with each parameter. Information can be fused in accordance with MYCIN certainty factor combination rule and the best alternatives are achieved. By using three examples, comparing with the mean potentiality approach and giving an application to medical diagnosis problems, the feasibility and effectiveness of the approach are demonstrated.
\end{abstract}

Keywords: Fuzzy soft set; Decision making; Grey relational analysis; MYCIN certainty factor.

\section{Introduction}

To solve complicated problems in economics, engineering, environmental science and social science, methods in classical mathematics are not always successful because of the existence of various types of uncertainties. There are several theories: probability theory, fuzzy set theory ${ }^{46}$, rough set theory 37 and interval mathematics which we can consider as mathematical tools to deal with uncertainties. But all these theories have their own limitations ${ }^{31}$ which present many difficulties. To overcome these difficulties, a new approach was proposed in ${ }^{31}$, which is called soft set theory for dealing with uncertainty.
Recently, there has been a rapid growth of interest in soft set theory. Maji et al. ${ }^{32,34}$ further discussed soft set theory and defined fuzzy soft sets by combining soft sets with fuzzy sets. The study of hybrid models combining soft sets or fuzzy soft sets with other mathematical structures and new operations is emerging as an active research topic of soft set theory ${ }^{10,19,45}$. Aktas et al. ${ }^{1}$ initiated soft groups. Jun et al. ${ }^{16,17}$ applied soft sets to BCK/BCIalgebras. Feng et al. ${ }^{10}$ defined soft semirings and established the connection between soft sets and semirings. Jiang et al. ${ }^{19}$ extended soft sets with description logics. Li et al. ${ }^{27,28}$ considered roughness of fuzzy soft sets and obtained the relationship

\footnotetext{
${ }^{*}$ Corresponding author: Zhaowen Li
} 
among soft sets, soft rough sets and topologies. Li et al. ${ }^{30}$ studied parameter reductions of soft coverings. Nowadays, soft set theory has been proved to be useful in many kinds of fields, such as rule mining ${ }^{15}$, simulation ${ }^{21}$, forecasting ${ }^{43}$, data analysis 51 and decision making $3,23,38$.

Maji et al. ${ }^{35}$ first applied soft sets to solve decision making problems with the help of rough set approaches. Chen et al. ${ }^{5}$ defined the parameters reduction of soft sets and discussed its application of decision making problem. Cağman et al. ${ }^{3,4}$ presented soft matrix theory and uni-int decision making approach, which selected a set of optimum elements from different alternatives. Roy et al. ${ }^{38}$ discussed score value as the evaluation basis to find an optimal choice object in fuzzy soft sets. Feng et al. ${ }^{9}$ pointed out the limitation of Roy's method and applied level soft sets to discuss fuzzy soft sets based on decision making. Jiang et al. ${ }^{18}$ presented an adjustable approach to intuitionistic fuzzy soft sets 33 based on decision making by using level soft sets of intuitionistic fuzzy soft sets. Basu et al. ${ }^{2}$ further compared the previous methods to fuzzy soft sets in decision making and introduced the mean potentiality approach, which showed more efficient and more accurate than the previous.

The unique or uniform criterion does not exist for the selection on the above approaches to fuzzy soft sets in decision making. Although researchers focus on a direction as the evaluation basis, such as choice value, score value or others, the same decision making problem may obtain different results from using various evaluation bases. As a result, it is difficult to judge which result is better and which method should be chosen for selecting the optimal choice object. The key to this problem is how to reduce subjectivity and uncertainty. Then it is necessary to pay attention to this issue.

Grey relational analysis method is important to reflect uncertainty in grey system theory initiated by Deng ${ }^{6}$, which is utilized for generalizing estimates under small samples and uncertain conditions. Liu ${ }^{24}$ further develop the theory. And it has been successfully applied in solving decision making problems $22,42,50$. Certainty factor has been proposed by Shortliffe and Buchanan ${ }^{39}$ and then applied in
MYICIN expert system ${ }^{40}$. Then Heckerman ${ }^{7}$ developed it. It is a powerful method for combining accumulative evidences of changing prior opinions in the light of new evidences. Compared to probability theory, MYCIN certainty factor captures more information to support decision making by identifying the uncertain and unknown evidence. It provides the mechanism to derive solutions from various vague evidences without knowing much prior information. The advantage of this model is more intuitive and easier to master, its algorithm is simpler and the less amount of calculation. Moreover, it avoids the prior probability or priori possibility problems in probability or possibility theory. MYCIN certainty factor has been successfully applied into many fields such as intelligent medical diagnosis, earthquake prediction and program design ${ }^{25,40}$. Decision makers are able to take full advantage of both methods' merits to deal with uncertainty and risk confidently by combining both above theories. Thus, it not only avoids the problem of selecting the suitable level soft sets to obtain choice values and score values, but also reduces uncertainty caused by people's subjective cognition so as to raise the choice decision level. And the application to medical diagnosis problems show that the hybrid model is effective and practical under uncertain conditions.

The remaining part of this paper is organized as follows. In Section 2, we present some concepts about fuzzy soft sets and MYCIN certainty factor. In Section 3, we recall the mean potentiality approach to fuzzy soft sets in decision making and give three examples to illustrate this approach. In Section 4, we apply grey relational analysis to determine the uncertain degree of each parameter, by which suitable essence uncertainty factor with respect to each parameter is constructed. And we do decision making by using MYCIN certainty factor combination rule. In Section 5, we give measure of performance and the interpretations of the results. In Section 6, we demonstrate the feasibility of new approach by comparing with the mean potentiality approach and giving an application to medical diagnosis problems. In Section 7, we end this paper with some conclusions. 


\section{Preliminaries}

Throughout this paper, $U$ denotes an initial universe, $E$ denotes the set of all possible parameters and $I^{U}$ denotes the set of all fuzzy subsets of $U$. We only consider the case where $U$ and $E$ are both nonempty finite sets.

In this section, we recall some basic concepts about fuzzy soft sets and MYCIN certainty factor.

\subsection{Fuzzy soft sets}

Definition 2.1 $\left({ }^{32}\right)$ Let $A \subseteq E . \quad A$ pair $(F, A)$ is called a fuzzy soft set over $U$, where $F$ is a mapping given by $F: A \rightarrow I^{U}$.

In other words, a fuzzy soft set is a parametrized family of fuzzy sets in the universe. For each $e \in A$. $F(e)$ is a fuzzy set in $U$ and is called a fuzzy set with respect to the parameter $e$.

Denote $U=\left\{h_{1}, h_{2}, \ldots, h_{m}\right\}$ and $A=$ $\left\{e_{1}, e_{2}, \ldots, e_{n}\right\}$. Put

$$
g_{i j}=F\left(e_{j}\right)\left(h_{i}\right) .
$$

Then $G=\left(g_{i j}\right)_{m \times n}$ is called the fuzzy soft matrix induced by $(F, A)$.

It is easy to see that every soft set may be considered as a fuzzy soft set ${ }^{9}$.

Example 2.2 Let $(F, A)$ be a fuzzy soft set over $U$ where $U=\left\{h_{1}, h_{2}, h_{3}, h_{4}, h_{5}\right\}$ and $A=$ $\left\{e_{1}, e_{2}, e_{3}, e_{4}, e_{5}, e_{6}\right\}$, defined as follows:

$$
\begin{aligned}
& F\left(e_{1}\right)=\frac{0.8}{h_{1}}+\frac{1}{h_{2}}+\frac{0.2}{h_{3}}+\frac{0.3}{h_{4}}+\frac{1}{h_{5}}, \\
& F\left(e_{2}\right)=\frac{0.5}{h_{1}}+\frac{0.1}{h_{2}}+\frac{0.3}{h_{3}}+\frac{0.2}{h_{4}}+\frac{0.1}{h_{5}}, \\
& F\left(e_{3}\right)=\frac{0.1}{h_{1}}+\frac{0.4}{h_{2}}+\frac{1}{h_{3}}+\frac{1}{h_{4}}+\frac{0.8}{h_{5}}, \\
& F\left(e_{4}\right)=\frac{0}{h_{1}}+\frac{0.3}{h_{2}}+\frac{0.1}{h_{3}}+\frac{1}{h_{4}}+\frac{0}{h_{5}}, \\
& F\left(e_{5}\right)=\frac{0.6}{h_{1}}+\frac{0}{h_{2}}+\frac{1}{h_{3}}+\frac{0.3}{h_{4}}+\frac{0.7}{h_{5}}, \\
& F\left(e_{6}\right)=\frac{0.1}{h_{1}}+\frac{0.4}{h_{2}}+\frac{0.7}{h_{3}}+\frac{1}{h_{4}}+\frac{0.2}{h_{5}} .
\end{aligned}
$$

Then $(F, A)$ is described by Table 1.
Table 1: Tabular representation of the fuzzy soft $\operatorname{set}(F, A)$

\begin{tabular}{lccccc}
\hline & $h_{1}$ & $h_{2}$ & $h_{3}$ & $h_{4}$ & $h_{5}$ \\
\hline$e_{1}$ & 0.8 & 1 & 0.2 & 0.3 & 1 \\
$e_{2}$ & 0.5 & 0.1 & 0.3 & 0.2 & 0.1 \\
$e_{3}$ & 0.1 & 0.4 & 1 & 1 & 0.8 \\
$e_{4}$ & 0 & 0.3 & 0.1 & 1 & 0 \\
$e_{5}$ & 0.6 & 0 & 1 & 0.3 & 0.7 \\
$e_{6}$ & 0.1 & 0.4 & 0.7 & 1 & 0.2 \\
\hline
\end{tabular}

If $h$ is viewed as an assumption(or object), then $F(e)(h)$ may represent the degree that the parameter $e$ holds the object $h$ and $F(e)^{c}(h)$ may represent the degree that the parameter $e$ opposes the object $h$.

Obviously, $F(e)(h)+F(e)^{c}(h)=1$.

Definition 2.3 Let $A \subseteq E$ and let $(F, A)$ be a fuzzy soft set over $U . S_{e}(h)=F(e)(h)-F(e)^{c}(h)$ is called the score function value of $h$ with respect to $e$.

The score function represents the difference between the support degree and the opposite degree. Especially, when $S_{e}(h)=1$, it expresses the parameter $e$ is all for the object $h ; S_{e}(h)=-1$, it expresses the parameter e totally against to the object $h ; S_{e}(h)=0$, it expresses the same degree of the parameter e support or opposite the object $h$.

Denote $U=\left\{h_{1}, h_{2}, \ldots, h_{m}\right\}$ and $A=$ $\left\{e_{1}, e_{2}, \ldots, e_{n}\right\}$. Put

$$
s_{i j}=F\left(e_{j}\right)\left(h_{i}\right)-F\left(e_{j}\right)^{c}\left(h_{i}\right) .
$$

Then $S=\left(s_{i j}\right)_{m \times n}$ is called the score matrix induced by $(F, A)$.

Definition 2.4 $\left({ }^{32}\right)$ Let $A, B \subseteq E$ and let $(F, A)$ and $(G, B)$ be two fuzzy soft sets over $U$. $(F, A)$ is a fuzzy soft subset of $(G, B)$ if

(i) $A \subseteq B$,

(ii) $F(e)$ is a fuzzy subset of $G(e)$ for any $e \in A$.

We denote it by $(F, A) \widetilde{\subset}(G, B)$ or $(G, B) \widetilde{\supset}(F, A)$, at this point, $(G, B)$ is said to be a fuzzy soft super set of $(F, A)$.

It is obvious that $(F, A)=(G, B)$ if and only if $(F, A) \widetilde{\subset}(G, B)$ and $(F, A) \widetilde{\supset}(G, B)$.

Definition $2.5\left({ }^{32}\right)$ Let $A, B \subseteq E$ and let $(F, A)$, $(G, B)$ be two fuzzy soft sets over $U$. Then " $(F, A)$ $A N D(G, B)$ " is a fuzzy soft set denoted by $(F, A) \wedge$ $(G, B)$ and is defined by $(F, A) \wedge(G, B)=(H, A \times B)$, where $H(\alpha, \beta)=F(\alpha) \cap G(\beta)$ for $\alpha \in A$ and $\beta \in B$, 
where $\cap$ is the operation "fuzzy intersection" of two fuzzy sets.

\subsection{MYCIN certainty factor}

Certainty factor's reliability is an imprecise reasoning model used by MYCIN system, it is a reasonable and effective inference model in many practical applications. MYCIN certainty factor is a new important inference method under uncertainty conditions. It has an advantage to deal with subjective judgments and to synthesize the uncertainty knowledge $^{49}$.

In this paper, $C F(e)$ represents the confidence degree of the evidence $e$ when the evidence $e$ is uncertain as true, its value is in $[-1,1]$. The larger value of $C F(e)$, the higher credibility of the evidence $e$. Specially, $C F(e)=1$ means the evidence $e$ is true; $C F(e)=-1$ means the evidence $e$ is fake.

Definition 2.6 $\left({ }^{49}\right)$ Let $h$ be a random variable of assumptions and let e be a random variable of evidences. Then $C F(h / e)=M B(h / e)-M D(h / e)$ is called MYCIN certainty factor, where

$$
\begin{gathered}
M B(h / e)= \begin{cases}\frac{P(h / e) \vee P(h)-P(h)}{1-P(h)}, & P(h) \neq 1 ; \\
1, & P(h)=1 ;\end{cases} \\
M D(h / e)= \begin{cases}\frac{P(h)-P(h / e) \wedge P(h)}{P(h)}, & P(h) \neq 0 ; \\
1, & P(h)=0 .\end{cases}
\end{gathered}
$$

$M B(h / e)$ is called the degree of confidence in growth which means the increase confidence level of the assumption $h$ at the emergence of evidence $e ; M D(h / e)$ is called the degree of no-confidence in growth which means the decrease confidence level of the assumption $h$ at the emergence of evidence $e$.

$C F(h / e)$ means the confidence degree of the assumption $h$ is true in case of the evidence $e$, its value is in $[-1,1]$. Especially, when $C F(h / e)=1$, it expresses assumption $h$ is true in case of the evidence $e ; C F(h / e)=-1$, it expresses assumption $h$ is fake in case of the evidence $e ; C F(h / e)=0$, it expresses assumption $h$ could not determine in case of the evidence $e$.
Definition $2.7\left({ }^{26}\right)$ Let $h$ be a random variable of assumption and let e be a random variable of evidence. If the evidence $e$ is uncertain as true, then the essence uncertainty factor is defined as:

$$
C F_{T}(h / e)=C F(h / e) \cdot C F(e) .
$$

$C F_{T}(h / e)$ represents the confidence degree of the assumption $h$ is true while the confidence degree of evidence e is $C F(e)$.

In reality, decision makers can often gain access to more than one information source for the sake of making decisions. Therefore, we introduce the following definitions. The generation function which can be synthesized constructs the synthetic function of the essence uncertainty factor from these information sources. And then we get the evidence synthesis formula. This construction is called MYCIN certainty factor combination rule for group aggregation.

Definition 2.8 $\left({ }^{49}\right.$ ) A function $F:[0, \infty) \rightarrow[-1,1]$ is called a generation function, if $F$ be satisfied the following two conditions:

(1) $F(0)=-1, F(\infty)=-1, F(x)$ is monotone increasing;

(2) $F(1 / x)=-F(x)$.

Definition 2.9 $\left({ }^{49}\right)$ The generation function $F$ : $[0, \infty) \rightarrow[-1,1]$ is called to be synthesized, if there is a function of two variables $f$ such that $F(x \cdot y)=$ $f(F(x), F(y))$.

Theorem 2.10 $\left({ }^{49}\right)$ Suppose that $e_{1}$ and $e_{2}$ are conditional independence about $h$ and $\bar{h}$. F is a generation function which can be synthesized. The generation function of the essence uncertainty factor is as follows:

$$
C F_{T}\left(h / e_{1}, e_{2}\right)=F\left(\frac{P\left(e_{1}, e_{2} / h\right)}{P\left(e_{1}, e_{2} / \bar{h}\right)}\right) .
$$

It means that there is a function $f$ : $[-1,1]^{2} \rightarrow[-1,1]$ such that $C_{T}\left(h / e_{1}, e_{2}\right)=$ $f\left(C F_{T}\left(h / e_{1}\right), C F_{T}\left(h / e_{2}\right)\right)$.

Because

$$
F_{1}(x \cdot y)=\frac{F_{1}(x)+F_{1}(y)}{1+F_{1}(x) \cdot F_{1}(y)}
$$

is a kind of generation function which can be synthesized, then the evidence synthesis formula is as follows: 


$$
C F_{T}\left(h / e_{1}, e_{2}\right)=\frac{C F_{T}\left(h / e_{1}\right)+C F_{T}\left(h / e_{2}\right)}{1+C F_{T}\left(h / e_{1}\right) \cdot C F_{T}\left(h / e_{2}\right)} .
$$

The synthesis of multiple evidences can be promoted according to MYCIN certainty factor combination rule:

Suppose that $e_{1}, e_{2}, \cdots, e_{m}$ are conditional independence about $h$ and $\bar{h} . F$ is a generation function which can be synthesized. The generation function of the essence uncertainty factor is as follows:

$$
C F_{T}\left(h / e_{1}, e_{2}, \cdots, e_{m}\right)=f\left(C F_{T}\left(h / e_{1}, e_{2}, \cdots, e_{m-1}\right), C F_{T}\left(h / e_{m}\right)\right) .
$$

That is,

$$
C F_{T}\left(h / e_{1}, e_{2}, \cdots, e_{m}\right)=\frac{C F_{T}\left(h / e_{1}, e_{2}, \cdots, e_{m-1}\right)+C F_{T}\left(h / e_{m}\right)}{1+C F_{T}\left(h / e_{1}, e_{2}, \cdots, e_{m-1}\right) \cdot C F_{T}\left(h / e_{m}\right)} .
$$

MYCIN certainty factor combination rule can increase the confidence degree and reduce the uncertain degree of the whole evidences to improve reliability.

Example 2.11 Let $E=\left\{e_{1}, e_{2}\right\}$ be the set of evidences. Suppose there are two different assumptions $h_{1}$ and $h_{2}$ over $E$, induced by an independent piece of evidences $e_{1}, e_{2}$, given by

$$
\begin{aligned}
& C F_{T}\left(h_{1} / e_{1}\right)=0.2, C F_{T}\left(h_{1} / e_{2}\right)=0.7, \\
& C F_{T}\left(h_{2} / e_{1}\right)=0.4, C F_{T}\left(h_{2} / e_{2}\right)=0.3 .
\end{aligned}
$$

Combining the two evidences by MYCIN certainty factor combination rule leads to:

$$
\begin{aligned}
C F_{T}\left(h_{1} / e_{1}, e_{2}\right) & =\frac{C F_{T}\left(h_{1} / e_{1}\right)+C F_{T}\left(h_{1} / e_{2}\right)}{1+C F_{T}\left(h_{1} / e_{1}\right) \cdot C F_{T}\left(h_{1} / e_{2}\right)} \\
& =\frac{0.2+0.7}{1+0.2 \times 0.7}=0.7895 \\
C F_{T}\left(h_{2} / e_{1}, e_{2}\right) & =\frac{C F_{T}\left(h_{2} / e_{1}\right)+C F_{T}\left(h_{2} / e_{2}\right)}{1+C F_{T}\left(h_{2} / e_{1}\right) \cdot C F_{T}\left(h_{2} / e_{2}\right)} \\
& =\frac{0.4+0.3}{1+0.4 \times 0.3}=0.4117 .
\end{aligned}
$$

\section{Mean potentiality approach}

Just as most of the decision making problems, fuzzy soft sets based on decision making involve the evaluation of all decision alternatives. Recently, applications of fuzzy soft sets based on decision making have attracted more and more attentions. The works of Roy et al. 9,20,38 are fundamental and significant. Later Kong et al. ${ }^{23}$ applied grey relational analysis to solve fuzzy soft sets in decision making. Generally, there does not exist any unique or uniform criterion for the evaluation of decision alternatives under uncertain conditions. Thus, Basu et al. ${ }^{2}$ further studied and proposed the mean potentiality approach to fuzzy soft sets in decision making, which is more deterministic and accurate than Feng's approach ${ }^{9}$.

Below, we introduce Basu's approach.

Let $U=\left\{h_{1}, h_{2}, \cdots, h_{m}\right\}$ be the universe and $A=$ $\left\{e_{1}, e_{2}, \cdots, e_{n}\right\}$ be a set of parameters. Given a fuzzy soft set $(F, A)$. We mainly recall the mean potentiality approach to $(F, A)$ based on decision making problems with equally weighted choice parameters:

Step 1. Find a normal parameter reduction $B$ of $A^{36}$. If it exists, we construct the tabular representation of $(F, B)$. Otherwise, we construct the tabular representation of $(F, A)$ with the choice values of each object.

Step 2. Compute the mean potentiality $m_{p}=$ $\frac{\sum_{i=1}^{m} \sum_{j=1}^{n} F\left(e_{j}\right)\left(h_{i}\right)}{m \times n}$ up to $\rho$ significant figures, denoted by $m_{p}^{\prime}$.

Step 3. Construct a $m_{p}^{\prime}$-level soft set of $(F, A)$ and represent it in tabular form, then compute the choice value $c_{i}$ for each $h_{i}$.

Step 4. Denote $\max \left\{c_{1}, c_{2}, \cdots, c_{m}\right\}=c_{k}$. If $c_{k}$ is unique, then the optimal choice object is $h_{k}$ and the process will be stopped. Otherwise, go to Step 5.

Step 5. Compute the non-negative difference between the largest and the smallest membership value in each column (resp. each row) and denote it as $\alpha_{j}$ $(j=1,2, \cdots, n)\left(\right.$ resp. $\left.\beta_{i}(i=1,2, \cdots, m)\right)$.

Step 6. Compute the average $\alpha=\frac{\sum_{j=1}^{n} a_{j}}{n}$ up to $\rho$ significant figures, denoted by $\alpha^{\prime}$.

Step 7. Construct a $\alpha^{\prime}$-level soft set of $(F, A)$ and represent it in tabular form, then compute the choice value $c_{i}^{\prime}$ for each $h_{i}$.

Step 8. Denote $\max \left\{c_{1}^{\prime}, c_{2}^{\prime}, \cdots, c_{m}^{\prime}\right\}=c_{l}^{\prime}$. If $c_{l}^{\prime}$ is unique, then the optimal choice object is $h_{l}$ and the process will be stopped. Otherwise, go to Step 9.

Step 9. Consider the object corresponding to the minimum value of $\beta_{i}(i=1,2, \cdots, m)$ as the optimal choice of decision makers.

The following examples illustrate the mean po- 
tentiality approach to fuzzy soft sets in decision making. Firstly, we give an example which can be stopped at Step 4.

Example 3.1 Let us consider a decision making problem which is associated with the fuzzy soft set $(F, A)$ given in Table 2.

(1) Since A is indispensable, there does not exist any normal parameter reduction of $A$.

(2) Since the mean potentiality of $(F, A)$ is $m_{p}=$ $\frac{\sum_{i=1}^{3} \sum_{j=1}^{3} F\left(e_{j}\right)\left(h_{i}\right)}{3 \times 3}=0.655$, then $m_{p}^{\prime}=0.6$.

(3) $m_{p}^{\prime}$-level soft set of $(F, A)$ with choice values is given by Table 3 .

(4) Since $\max \left\{c_{i}, i=1,2,3\right\}=c_{2}$, then the optimal choice is $h_{2}$.

Table 2: Tabular representation of $(F, A)$

\begin{tabular}{lccc}
\hline & $e_{1}$ & $e_{2}$ & $e_{3}$ \\
\hline$h_{1}$ & 0.8 & 0.7 & 0.2 \\
$h_{2}$ & 0.6 & 0.9 & 0.7 \\
$h_{3}$ & 0.7 & 0.5 & 0.8 \\
\hline
\end{tabular}

Table 3: Tabular representation of $L((F, A), 0.6)$ with choice values

\begin{tabular}{ccccc}
\hline & $e_{1}$ & $e_{2}$ & $e_{3}$ & Choice value \\
\hline$h_{1}$ & 1 & 1 & 0 & 2 \\
$h_{2}$ & 1 & 1 & 1 & 3 \\
$h_{3}$ & 1 & 0 & 1 & 2 \\
\hline
\end{tabular}

Secondly, we give another example which can be stopped at Step 9.

Example 3.2 Let us consider a decision making problem which is associated with the fuzzy soft set $(F, A)$ given in Table 4.

(1) Since A is indispensable, there does not exist any normal parameter reduction of $A$.

(2) Since the mean potentiality of $(F, A)$ is $m_{p}=$ $\frac{\sum_{i=1}^{3} \sum_{j=1}^{5} F\left(e_{j}\right)\left(h_{i}\right)}{3 \times 5}=0.622$, then $m_{p}^{\prime}=0.62$.

(3) $m_{p}^{\prime}$-level soft set of $(F, A)$ with choice values is given by Table 5 .

(4) Since $h_{1}$ and $h_{2}$ have the same maximum choice values (3), we have to calculate the $\alpha_{j}$ and $\beta_{i}$ values of $(F, A)$.

(5) See Table 6.

(6) Now $\alpha=\frac{0.29+0.27+0.56+0.32+0.32}{5}=0.352$, thus $\alpha^{\prime}=0.35$.

(7) So the $\alpha^{\prime}$-level soft set of $(F, A)$ with choice values $c_{i}^{\prime}(i=1,2,3)$ is given by Table 7 .
(8) Here $\max \left\{c_{1}^{\prime}, c_{2}^{\prime}, c_{3}^{\prime}\right\}=\left\{c_{2}^{\prime}, c_{3}^{\prime}\right\}$ (i.e., not unique), we have to consider the $\beta_{2}$ and $\beta_{3}$.

(9) Since $\min \left\{\beta_{2}, \beta_{3}\right\}=\beta_{3}(=0.37)$, then $h_{3}$ is the optimal choice object.

Table 4: Tabular representation of $(F, A)$

\begin{tabular}{cccccc}
\hline & $e_{1}$ & $e_{2}$ & $e_{3}$ & $e_{4}$ & $e_{5}$ \\
\hline$h_{1}$ & 0.85 & 0.73 & 0.26 & 0.32 & 0.75 \\
$h_{2}$ & 0.56 & 0.82 & 0.76 & 0.64 & 0.43 \\
$h_{3}$ & 0.84 & 0.55 & 0.82 & 0.53 & 0.47 \\
\hline
\end{tabular}

Table 5: Tabular representation of $L((F, A), 0.62)$ with choice values

\begin{tabular}{ccccccc}
\hline & $e_{1}$ & $e_{2}$ & $e_{3}$ & $e_{4}$ & $e_{5}$ & Choice value \\
\hline$h_{1}$ & 1 & 1 & 0 & 0 & 1 & 3 \\
$h_{2}$ & 0 & 1 & 1 & 1 & 0 & 3 \\
$h_{3}$ & 1 & 0 & 1 & 0 & 0 & 2 \\
\hline
\end{tabular}

Table 6: Tabular representation of $(F, A)$ with $\alpha_{i}$ and $\beta_{j}$ values

\begin{tabular}{ccccccc}
\hline & $e_{1}$ & $e_{2}$ & $e_{3}$ & $e_{4}$ & $e_{5}$ & $\beta_{i}$ \\
\hline$h_{1}$ & 0.85 & 0.73 & 0.26 & 0.32 & 0.75 & 0.59 \\
$h_{2}$ & 0.56 & 0.82 & 0.76 & 0.64 & 0.43 & 0.39 \\
$h_{3}$ & 0.84 & 0.55 & 0.82 & 0.53 & 0.47 & 0.37 \\
$\alpha_{i}$ & 0.29 & 0.27 & 0.56 & 0.32 & 0.32 & \\
\hline
\end{tabular}

Table 7: Tabular representation of $L((F, A), 0.35)$ with choice values

\begin{tabular}{ccccccc}
\hline & $e_{1}$ & $e_{2}$ & $e_{3}$ & $e_{4}$ & $e_{5}$ & Choice value \\
\hline$h_{1}$ & 1 & 1 & 0 & 0 & 1 & 3 \\
$h_{2}$ & 1 & 1 & 1 & 1 & 1 & 5 \\
$h_{3}$ & 1 & 1 & 1 & 1 & 1 & 5
\end{tabular}

We can apply the mean potentiality approach to deal with the above two cases. Now, we consider the following example.

Example 3.3 Let us consider a decision making problem which is associated with the fuzzy soft set $(F, A)$ given in Table 8.

(1) Since A is indispensable, there does not exist any normal parameter reduction of $A$.

(2) Since the mean potentiality of $(F, A)$ is $m_{p}=$ $\frac{\sum_{i=1}^{3} \sum_{j=1}^{5} F\left(e_{j}\right)\left(h_{i}\right)}{3 \times 5}=0.7455$, then $m_{p}^{\prime}=0.74$.

(3) $m_{p}^{\prime}$-level soft set of $(F, A)$ with choice values is given by Table 9.

(4) Since $h_{1}$ and $h_{2}$ have the same maximum choice values (3), we have to calculate the $\alpha_{j}$ and $\beta_{i}$ values of $(F, A)$.

(5) See Table 10 

0.33 .

(6) Now $\alpha=\frac{0.2+0.4+0.4}{3}=0.3333$, thus $\alpha^{\prime}=$

(7) So the $\alpha^{\prime}$-level soft set of $(F, A)$ with choice values $c_{i}^{\prime}(i=1,2,3)$ is given by Table 11 .

(8) Here $\max \left\{c_{1}^{\prime}, c_{2}^{\prime}, c_{3}^{\prime}\right\}=\left\{c_{2}^{\prime}, c_{3}^{\prime}\right\}$ (i.e., not unique), we have to consider the $\beta_{2}$ and $\beta_{3}$.

(9) Since $\beta_{2}=\beta_{3}(=0.30), h_{2}$ or $h_{3}$ is the optimal choice object.

Table 8: Tabular representation of $(F, A)$

\begin{tabular}{cccc}
\hline & $e_{1}$ & $e_{2}$ & $e_{3}$ \\
\hline$h_{1}$ & 0.89 & 0.79 & 0.29 \\
$h_{2}$ & 0.69 & 0.99 & 0.79 \\
$h_{3}$ & 0.79 & 0.59 & 0.89 \\
\hline
\end{tabular}

Table 9: Tabular representation of $L((F, A), 0.74)$ with choice values

\begin{tabular}{ccccc}
\hline & $e_{1}$ & $e_{2}$ & $e_{3}$ & Choice value \\
\hline$h_{1}$ & 1 & 1 & 0 & 2 \\
$h_{2}$ & 0 & 1 & 1 & 2 \\
$h_{3}$ & 1 & 0 & 1 & 2 \\
\hline
\end{tabular}

Table 10: Tabular representation of $(F, A)$ with $\alpha_{i}$ and $\beta_{j}$ values

\begin{tabular}{ccccc}
\hline & $e_{1}$ & $e_{2}$ & $e_{3}$ & $\beta_{i}$ \\
\hline$h_{1}$ & 0.89 & 0.79 & 0.29 & 0.60 \\
$h_{2}$ & 0.69 & 0.99 & 0.79 & 0.30 \\
$h_{3}$ & 0.79 & 0.59 & 0.89 & 0.30 \\
$\alpha_{i}$ & 0.2 & 0.4 & 0.4 & \\
\hline
\end{tabular}

Table 11: Tabular representation of $L((F, A), 0.33)$ with choice values

\begin{tabular}{ccccc}
\hline & $e_{1}$ & $e_{2}$ & $e_{3}$ & Choice value \\
\hline$h_{1}$ & 1 & 1 & 0 & 2 \\
$h_{2}$ & 1 & 1 & 1 & 3 \\
$h_{3}$ & 1 & 1 & 1 & 3 \\
\hline
\end{tabular}

By Basu's approach, decision makers can not decide which one is the optimal choice object. Now we try to overcome this problem by proposing a new approach for decision making problem of fuzzy soft sets as follows.

\section{A novel approach to fuzzy soft sets in decision making based on grey relational analysis and MYCIN certainty factor}

The existing approaches to fuzzy soft sets in decision making are mainly based on the level soft set to obtain useful information such as choice values and score values. However, it is very difficult for decision makers to select a suitable level soft set. Now we introduce a new approach to fuzzy soft sets in decision making based on grey relational analysis and MYCIN certainty factor. The approach is effective and practical under uncertain conditions. It not only allows us to avoid the problem of selecting the suitable level soft set, but also helps reducing uncertainty caused by people's subjective cognition so as to raise the choice decision level.

This approach include three phases: First, grey relational analysis is applied to calculate the grey mean relational degree between each independent alternative and the mean of all alternatives with each parameter, and the uncertain degree of each parameter is obtained. Second, the suitable essence uncertainty factor with respect to each parameter (or evidence) is constructed by the uncertain degree of each parameter. Third, we apply MYCIN certainty factor combination rule to aggregate independent evidences into a collective evidence, by which the candidate alternatives are ranked and the best alternative(s) are obtained. The stepwise procedure of this approach is described in Fig. 1.

\subsection{The approach}

In this paper, regard the solution system of decision making system as a set of different assumptions and treat the target system as a set of evidences.

In the following, we consider a decision making problem concerned with $m$ different assumptions and $n$ different evidences (or parameters), and we always denote

$$
U=\left\{h_{1}, h_{2}, \cdots, h_{m}\right\}, A=\left\{e_{1}, e_{2}, \cdots, e_{n}\right\} .
$$

Let $(F, A)$ be a fuzzy soft set over $U$ and let $G=\left(g_{i j}\right)_{m \times n}$ be the fuzzy soft matrix induced by $(F, A)$.

Compared to the score function $S_{e}(h)$ and MYCIN certainty factor $C F(h / e)$, both are very similar in the sense, so we can substitute $S_{e}(h)$ for $C F(h / e)$, it means that $C F\left(h_{i} / e_{j}\right)=s_{i j}$.

Compared to probability theory, MYCIN certainty factor captures more information to support decision making, by identifying the uncertain and 


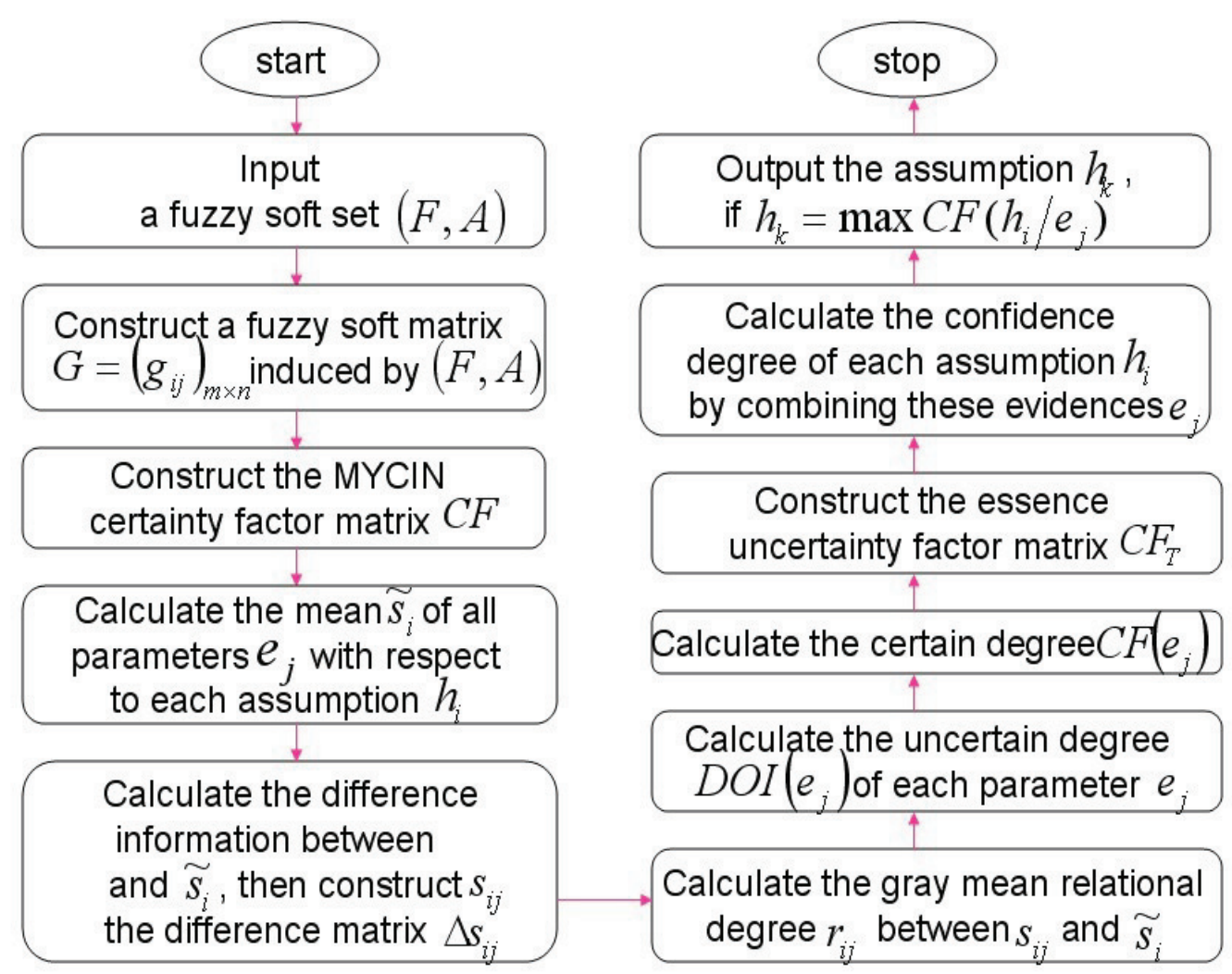

Figure 1: Flow chart of algorithm.

unknown evidences. It provides a mechanism to derive solutions from various vague evidences without knowing much prior information. The primary problem is how to obtain the uncertain degree of evidences.

First we present some basic notions.

Definition 4.1 $\left({ }^{41}\right)$ Let $(F, A)$ be a fuzzy soft set over $U$. Suppose that $G=\left(g_{i j}\right)_{m \times n}$ and $S=\left(s_{i j}\right)_{m \times n}$ are respectively the fuzzy soft matrix and the score matrix induced by $(F, A)$. For any $i, j$, denote

$$
\begin{gathered}
\widetilde{s_{i}}=\frac{1}{n} \sum_{j=1}^{n} s_{i j}, \quad \triangle s_{i j}=\left|s_{i j}-\widetilde{s}_{i}\right|, \\
r_{i j}=\frac{\min _{1 \leqslant i \leqslant m} \triangle s_{i j}+\rho \max _{1 \leqslant i \leqslant m} \triangle s_{i j}}{\triangle s_{i j}+\rho \max _{1 \leqslant i \leqslant m} \triangle s_{i j}}, \\
\text { where } \rho \in(0,1),
\end{gathered}
$$

$$
\operatorname{DOI}\left(e_{j}\right)=\frac{1}{m}\left(\sum_{i=1}^{m}\left(r_{i j}\right)^{q}\right)^{\frac{1}{q}} .
$$

Then

(1) $\widetilde{s_{i}}$ is called the mean of all parameters with respect to $h_{i}$,

(2) $\triangle s_{i j}$ is called the difference information between $s_{i j}$ and $\widetilde{s_{i}}$,

(3) $\rho$ is called the distinguishing coefficient and $r_{i j}$ is called the grey mean relational degree between $s_{i j}$ and $\widetilde{s}_{i}$,

(4) DOI $\left(e_{j}\right)$ is called the q order uncertain degree of the parameter $e_{j}$.

In this paper we pick $\rho=0.5, q=2$ and just consider grey mean relational degree between $s_{i j}$ and $\widetilde{s_{i}}$ 29.

MYCIN certainty factor is a powerful tool for combining accumulative evidences of changing prior opinions in the light of new evidences ${ }^{49}$. The 
primary procedure about combining the known evidences or information with other evidences is to construct suitable essence uncertainty factor. It is flexible to obtain MYCIN certainty factor. People's experience, knowledge or thinking will affect the selection of MYCIN certainty factor.

Now, by the uncertain degree of each parameter, we can obtain production function of each assumption with respect to each parameter.

Definition 4.2 Let $(F, A)$ be a fuzzy soft set over $U$. Suppose that $G=\left(g_{i j}\right)_{m \times n}$ and $S=\left(s_{i j}\right)_{m \times n}$ are respectively the fuzzy soft matrix and score matrix induced by $(F, A)$.

Put

$$
\begin{gathered}
C F\left(e_{j}\right)=1-\operatorname{DOI}\left(e_{j}\right), \\
C F_{T}\left(h_{i} / e_{j}\right)=C F\left(h_{i} / e_{j}\right) \cdot C F\left(e_{j}\right) \\
=s_{i j}\left(1-\operatorname{DOI}\left(e_{j}\right)\right), \\
C F=\left(C F\left(h_{i} / e_{j}\right)\right)_{m \times n}, \\
C F_{T}=\left(C F_{T}\left(h_{i} / e_{j}\right)\right)_{m \times n} .
\end{gathered}
$$

Then $C F\left(e_{j}\right)$ is called the certain degree of the parameter $e_{j}, C F_{T}\left(h_{i} / e_{j}\right)$ is called the essence uncertainty factor of the assumption $h_{i}$ in case of the evidence $e_{j}$, and $C F$ and $C F_{T}$ are respectively called the MYCIN certainty factor matrix and essence uncertainty factor matrix induced by $(F, A)$.

Based on the above analysis, an approach to the fuzzy soft set $(F, A)$ in decision making based on grey relational analysis and MYCIN certainty factor can be summarized as follows:

Step 1. Construct the fuzzy soft matrix $G=$ $\left(g_{i j}\right)_{m \times n}$ induced by $(F, A)$.

Step 2. Construct the soft matrix $S=\left(s_{i j}\right)_{m \times n}$ induced by $(F, A)$ and the MYCIN certainty factor matrix $C F$ induced by $(F, A)$.

Step 3. Calculate the mean of all parameters with respect to each assumption by

$$
\widetilde{s_{i}}=\frac{1}{n} \sum_{j=1}^{n} s_{i j} .
$$

Step 4. Calculate the difference information between $s_{i j}$ and $\widetilde{s_{i}}$ and construct the difference matrix by

$$
\triangle s_{i j}=\left|s_{i j}-\widetilde{s_{i}}\right|, \quad \triangle S=\left(\triangle s_{i j}\right)_{m \times n} .
$$

Step 5. Calculate the gray mean relational degree between $s_{i j}$ and $\widetilde{s_{i}}$ by

$$
r_{i j}=\frac{\min _{1 \leqslant i \leqslant m} \triangle s_{i j}+0.5 \max _{1 \leqslant i \leqslant m} \triangle s_{i j}}{\triangle s_{i j}+0.5 \max _{1 \leqslant i \leqslant m} \triangle s_{i j}} .
$$

Step 6. Calculate the uncertain degree of the parameter $e_{j}$ by

$$
\operatorname{DOI}\left(e_{j}\right)=\frac{1}{m}\left(\sum_{i=1}^{m}\left(r_{i j}\right)^{2}\right)^{\frac{1}{2}} .
$$

Step 7. Calculate the certain degree $C F\left(e_{j}\right)$ of the parameter $e_{j}$ by Definition 4.2.

Step 8. Construct the essence uncertainty factor matrix $C F_{T}$ induced by $(F, A)$.

Step 9. Calculate the confidence degree of each assumption $h_{i}$ by combining these evidences respectively by Theorem 2.10. Obtain decision making. The decision is $h_{k}$ if $\left.h_{k}=\max C F_{T}\left(h_{i} / e_{j}\right)\right)$. Optimal choices have more than one object if there are more assumptions corresponding to the maximum.

\subsection{Example illustration}

In this subsection, we give the following examples to illustrate new approach to fuzzy soft sets in decision making.

Example 4.3 Using new approach, we reconsider the fuzzy soft set $(F, A)$ given in Examples 3.1.

We suppose that the universe $U=\left\{h_{1}, h_{2}, h_{3}\right\}$ as a set of different assumptions and the set of parameters $A=\left\{e_{1}, e_{2}, e_{3}\right\}$ as a set of evidences. The process is as follows:

(1) Construct the fuzzy soft matrix $G=\left(g_{i j}\right)_{m \times n}$ induced by $(F, A)$ as follows:

$$
G=\left(g_{i j}\right)_{3 \times 3}=\left(\begin{array}{lll}
0.8 & 0.7 & 0.2 \\
0.6 & 0.9 & 0.7 \\
0.7 & 0.5 & 0.8
\end{array}\right) .
$$

(2) Construct the MYCIN certainty factor matrix as follows:

$$
C F=\left(s_{i j}\right)_{3 \times 3}=\left(\begin{array}{ccc}
0.6 & 0.4 & -0.6 \\
0.2 & 0.8 & 0.4 \\
0.4 & 0 & 0.6
\end{array}\right) .
$$


(3) Calculate the mean of all parameters of each assumption $h_{i}$ as follows:

$$
\widetilde{s_{1}}=0.1333, \widetilde{s_{2}}=0.4667, \widetilde{s_{3}}=0.3333 .
$$

(4) Calculate the difference information between $s_{i j}$ and $\widetilde{s}_{i}$, and construct the difference matrix as follows:

$$
\triangle S=\left(\begin{array}{lll}
0.4667 & 0.2667 & 0.7333 \\
0.2667 & 0.3333 & 0.0667 \\
0.0667 & 0.3333 & 0.2667
\end{array}\right)
$$

(5) Calculate the gray mean relational degree between $s_{i j}$ and $\widetilde{s_{i}}$ based on $\triangle S$ as follows:

$$
\left(r_{i j}\right)_{3 \times 5}=\left(\begin{array}{lll}
0.4286 & 1.0000 & 0.3939 \\
0.6000 & 0.8667 & 1.0000 \\
1.0000 & 0.8667 & 0.6842
\end{array}\right) \text {. }
$$

(6) Calculate the uncertain degree of each parameter $e_{j}$ as follows:

$$
\begin{aligned}
& \operatorname{DOI}\left(e_{1}\right)=0.4141, \\
& \operatorname{DOI}\left(e_{2}\right)=0.5273, \\
& \operatorname{DOI}\left(e_{3}\right)=0.4247 .
\end{aligned}
$$

(7) Calculate the certain degree of each parameter $e_{j}$ as follows:

$$
\begin{aligned}
& C F\left(e_{1}\right)=0.5859, \\
& C F\left(e_{2}\right)=0.4727, \\
& C F\left(e_{3}\right)=0.5753 .
\end{aligned}
$$

(8) Construct the essence uncertainty factor matrix $\mathrm{CF}_{T}$ as follows:

$$
C F_{T}=\left(\begin{array}{ccc}
0.3515 & 0.1891 & -0.3452 \\
0.1172 & 0.3782 & 0.2301 \\
0.2343 & 0 & 0.3452
\end{array}\right)
$$

(9) Calculate the confidence degree of each assumption $h_{i}$ by combining these evidences respectively as follows:

$$
C F_{T}\left(h_{1} / e_{1}, e_{2}, e_{3}\right)=0.1960,
$$

$$
\begin{aligned}
& C F_{T}\left(h_{2} / e_{1}, e_{2}, e_{3}\right)=0.6351, \\
& C F_{T}\left(h_{3} / e_{1}, e_{2}, e_{3}\right)=0.5362 .
\end{aligned}
$$

Then the final rang order is $h_{2} \succ h_{3} \succ h_{1}$. This consequence is the same as the one of Basu's approach.

Example 4.4 Using new approach, we reconsider the fuzzy soft set $(F, A)$ given in Examples 3.2.

We suppose that the universe $U=\left\{h_{1}, h_{2}, h_{3}\right\}$ as a set of different assumptions and the set of parameters $A=\left\{e_{1}, e_{2}, e_{3}, e_{4}, e_{5}\right\}$ as a set of evidences. The process is as follows:

(1) Construct the fuzzy soft matrix $G=\left(g_{i j}\right)_{m \times n}$ induced by $(F, A)$ as follows:

$$
G=\left(g_{i j}\right)_{3 \times 3}=\left(\begin{array}{ccccc}
0.85 & 0.73 & 0.26 & 0.32 & 0.75 \\
0.56 & 0.82 & 0.76 & 0.64 & 0.43 \\
0.84 & 0.55 & 0.82 & 0.53 & 0.47
\end{array}\right)
$$

(2) Construct the MYCIN certainty factor matrix as follows:

$$
C F=\left(s_{i j}\right)_{3 \times 5}=\left(\begin{array}{ccccc}
0.70 & 0.46 & -0.48 & -0.36 & 0.50 \\
0.12 & 0.64 & 0.52 & 0.28 & -0.14 \\
0.68 & 0.10 & 0.64 & 0.06 & -0.06
\end{array}\right)
$$

(3) Calculate the mean of all parameters of each assumption $h_{i}$ as follows:

$$
\widetilde{s_{1}}=0.1640, \widetilde{s_{2}}=0.2840, \widetilde{s_{3}}=0.2840 .
$$

(4) Calculate the difference information between $s_{i j}$ and $\widetilde{s_{i}}$, and construct the difference matrix as follows:

$$
\triangle S=\left(\begin{array}{lllll}
0.5360 & 0.2960 & 0.6440 & 0.5240 & 0.3360 \\
0.1640 & 0.3560 & 0.2360 & 0.0040 & 0.4240 \\
0.3960 & 0.1840 & 0.3560 & 0.2240 & 0.3440
\end{array}\right)
$$

(5) Calculate the gray mean relational degree between $s_{i j}$ and $\widetilde{s_{i}}$ based on $\triangle S$ as follows:

$$
\left(r_{i j}\right)_{3 \times 5}=\left(\begin{array}{lllll}
0.5373 & 0.7637 & 0.5776 & 0.3384 & 1.0000 \\
1.0000 & 0.6779 & 1.0000 & 1.0000 & 0.8616 \\
0.6506 & 1.0000 & 0.8230 & 0.5473 & 0.9856
\end{array}\right) \text {. }
$$

(6) Calculate the uncertain degree of each parameter $e_{j}$ as follows:

$$
\begin{gathered}
\operatorname{DOI}\left(e_{1}\right)=0.4361, \quad \operatorname{DOI}\left(e_{2}\right)=0.4764 \\
\operatorname{DOI}\left(e_{3}\right)=0.4727, \quad \operatorname{DOI}\left(e_{4}\right)=0.3964 \\
\operatorname{DOI}\left(e_{5}\right)=0.5491 .
\end{gathered}
$$


(7) Calculate the certain degree of each parameter $e_{j}$ as follows:

$$
\begin{gathered}
C F\left(e_{1}\right)=0.5639, \quad C F\left(e_{2}\right)=0.5236, \\
C F\left(e_{3}\right)=0.5273 . \quad C F\left(e_{4}\right)=0.6036, \\
C F\left(e_{5}\right)=0.4509 .
\end{gathered}
$$

(8) Construct the essence uncertainty factor matrix as follows:

$$
C F_{T}=\left(\begin{array}{ccccc}
0.3947 & 0.2408 & -0.2531 & -0.2173 & 0.2254 \\
0.0677 & 0.3351 & 0.2742 & 0.1690 & -0.0631 \\
0.3834 & 0.0524 & 0.3375 & 0.0362 & -0.0271
\end{array}\right) .
$$

(9) Calculate the confidence degree of each assumption $h_{i}$ by combining these evidences respectively as follows:

$$
\begin{aligned}
& C F_{T}\left(h_{1} / e_{1}, e_{2}, e_{3}\right)=0.3909, \\
& C F_{T}\left(h_{2} / e_{1}, e_{2}, e_{3}\right)=0.6669, \\
& C F_{T}\left(h_{3} / e_{1}, e_{2}, e_{3}\right)=0.6734 .
\end{aligned}
$$

Then the final rang order is $h_{3} \succ h_{2} \succ h_{1}$.This consequence is also the same as the one of Basu's approach.

Examples 4.3 and 4.4 illustrate that new approach is reasonable.

To address the issue in Example 3.3 we apply new approach. Matching process is as follows:

Example 4.5 We suppose that the universe $U=$ $\left\{h_{1}, h_{2}, h_{3}\right\}$ as a set of different assumptions and the set of parameters $A=\left\{e_{1}, e_{2}, e_{3}\right\}$ as a set of evidences. The process is as follows:

(1) Construct the fuzzy soft matrix $G=\left(g_{i j}\right)_{m \times n}$ induced by $(F, A)$.

$$
G=\left(g_{i j}\right)_{3 \times 3}=\left(\begin{array}{ccc}
0.89 & 0.79 & 0.29 \\
0.69 & 0.99 & 0.79 \\
0.79 & 0.59 & 0.89
\end{array}\right)
$$

(2) Construct the MYCIN certainty factor matrix induced by $(F, A)$.

$$
C F=\left(s_{i j}\right)_{3 \times 5}=\left(\begin{array}{ccc}
0.78 & 0.58 & -0.42 \\
0.38 & 0.98 & 0.58 \\
0.58 & 0.18 & 0.7800
\end{array}\right) \text {. }
$$

(3) Calculate the mean of all parameters of each assumption $h_{i}$ as follows:

$$
\widetilde{s_{1}}=0.3133, \widetilde{s_{2}}=0.6467, \widetilde{s_{3}}=0.5133,
$$

(4) Calculate the difference information between $s_{i j}$ and $\widetilde{s}_{i}$, and construct the difference matrix as follows:

$$
\triangle S=\left(\begin{array}{lll}
0.4667 & 0.2667 & 0.7333 \\
0.2667 & 0.3333 & 0.0667 \\
0.0667 & 0.3333 & 0.2667
\end{array}\right)
$$

(5) Calculate the gray mean relational degree between $s_{i j}$ and $\widetilde{s_{i}}$ based on $\triangle S$ as follows:

$$
\left(r_{i j}\right)_{3 \times 5}=\left(\begin{array}{ccc}
0.4286 & 1.0000 & 0.3939 \\
0.6000 & 0.8667 & 1.0000 \\
1.0000 & 0.8667 & 0.6842
\end{array}\right) .
$$

(6) Calculate the uncertain degree of each parameter $e_{j}$ as follows:

$$
\begin{aligned}
& \operatorname{DOI}\left(e_{1}\right)=0.4141, \\
& \operatorname{DOI}\left(e_{2}\right)=0.5273, \\
& \operatorname{DOI}\left(e_{3}\right)=0.4247,
\end{aligned}
$$

(7) Calculate the certain degree of each parameter $e_{j}$ as follows:

$$
\begin{aligned}
& C F\left(e_{1}\right)=0.5859, \\
& C F\left(e_{2}\right)=0.4727, \\
& C F\left(e_{3}\right)=0.5753 .
\end{aligned}
$$

(8) Construct the essence uncertainty factor matrixinduced by $(F, A)$.

$$
C F_{T}=\left(\begin{array}{ccc}
0.4570 & 0.2742 & -0.2416 \\
0.2226 & 0.4633 & 0.3337 \\
0.3398 & 0.0851 & 0.4487
\end{array}\right) \text {. }
$$

(9) Calculate the confidence degree of each assumption $h_{i}$ by combining these evidences respectively as follows: 


$$
\begin{aligned}
& C F_{T}\left(h_{1} / e_{1}, e_{2}, e_{3}\right)=0.4841, \\
& C F_{T}\left(h_{2} / e_{1}, e_{2}, e_{3}\right)=0.7913, \\
& C F_{T}\left(h_{3} / e_{1}, e_{2}, e_{3}\right)=0.7270 .
\end{aligned}
$$

Then the final rang order is $h_{2} \succ h_{3} \succ h_{1}$. Considering the former examples, we can push out this consequence is reasonable.

\section{Measure of performance and the interpretations of the results}

Definition $5.1\left({ }^{2}\right)$ Let $M$ be a method which satisfies n parameters optimality criteria based on fuzzy soft set. Let $F\left(e_{i}\right)\left(O_{p}\right)$ be the degree of the optimal object $O_{p}$ support the parameters $e_{i}$. Let

$$
\begin{gathered}
\zeta_{j k}^{i}=\left|F\left(e_{i}\right)\left(O_{p}\right)-F\left(e_{j}\right)\left(O_{p}\right)\right| \\
\Upsilon_{M}=\frac{1}{\sum_{i=1}^{n} \sum_{j=1, i \neq j}^{n} \zeta_{j k}^{i}}+\sum_{i=1}^{n} F\left(e_{i}\right)\left(O_{p}\right),
\end{gathered}
$$

$\zeta_{j k}^{i}$ is called the non-negative differences between the parameters $e_{i}$ and the parameters $e_{j}, \Upsilon_{M}$ is called the measure of performance of method $M$.

Let $M_{1}$ and $M_{2}$ be two methods which satisfy the optimality criteria based on fuzzy soft set, their measure of performances are $\Upsilon_{M_{1}}$ and $\Upsilon_{M_{2}}$. Now, if $\Upsilon_{M_{1}}>\Upsilon_{M_{2}}$ then method $\Upsilon_{M_{1}}$ is better than $\Upsilon_{M_{2}}$; on the contrary, method $\Upsilon_{M_{2}}$ is better than $\Upsilon_{M_{1}}$; if $\Upsilon_{M_{1}}=\Upsilon_{M_{2}}$ then the two method have the same performance.

We have gain the substantial uncertainty factors of assumption $h_{i}$ through the above analysis, as for the complexity of objective facts and the limitation of people's realization, for the problems in reality, there is overall uncertainty condition, so this paper propose a method to obtain the substantial uncertainty factors of overall uncertainty.

Considering that the substantial uncertainty factors of each assumption $h_{i}$ are induced by fuzzy soft set $(F, A)$. Then we can define the un-belief degree of real assumption $h_{i}$ as follows:

$$
\begin{aligned}
& C F_{T}^{c}\left(h_{i} / e_{1}, e_{2}, \cdots, e_{j}\right) \\
& \quad= \begin{cases}1-C F_{T}\left(h_{i} / e_{1}, e_{2}, \cdots, e_{j}\right), & C F_{T}\left(h_{i} / e_{1}, e_{2}, \cdots, e_{j}\right) \geqslant 0 ; \\
1+C F_{T}\left(h_{i} / e_{1}, e_{2}, \cdots, e_{j}\right), & C F_{T}\left(h_{i} / e_{1}, e_{2}, \cdots, e_{j}\right)<0 .\end{cases}
\end{aligned}
$$

The uncertainty degree of $h_{i}$ after evidence synthesis is

$C F_{U}\left(h_{i} / e_{1}, e_{2}, \ldots, e_{j}\right)=C F_{T}\left(h_{i} / e_{1}, e_{2}, \ldots, e_{j}\right)-C F_{T}^{c}\left(h_{i} / e_{1}, e_{2}, \ldots, e_{j}\right)$.

\section{Definition 5.2}

$$
C F_{U}=\frac{1}{m} \sum_{i=1}^{m} C F_{U}\left(h_{i} / e_{1}, e_{2}, \ldots, e_{j}\right)
$$

is called the substantial uncertainty factors of overall uncertainty.

Consider Example 3.3. We have

$$
\begin{aligned}
& C F_{T}\left(h_{1} / e_{1}, e_{2}, e_{3}\right)=0.1960, \\
& C F_{T}\left(h_{2} / e_{1}, e_{2}, e_{3}\right)=0.6351, \\
& C F_{T}\left(h_{3} / e_{1}, e_{2}, e_{3}\right)=0.5362 ; \\
& C F_{T}^{c}\left(h_{1} / e_{1}, e_{2}, e_{3}\right)=0.8040, \\
& C F_{T}^{c}\left(h_{2} / e_{1}, e_{2}, e_{3}\right)=0.3649, \\
& C F_{T}^{c}\left(h_{3} / e_{1}, e_{2}, e_{3}\right)=0.4638 .
\end{aligned}
$$

Calculate

$$
\begin{gathered}
C F_{U}\left(h_{1} / e_{1}, e_{2}, e_{3}\right)=-0.6080 \\
C F_{U}\left(h_{2} / e_{1}, e_{2}, e_{3}\right)=0.2702 \\
C F_{U}\left(h_{3} / e_{1}, e_{2}, e_{3}\right)=0.0724
\end{gathered}
$$

Then

$$
\frac{1}{3} \sum_{i=1}^{3} C F_{U}\left(h_{i} / e_{1}, e_{2}, e_{3}\right)=0.3168 .
$$

Calculate the substantial uncertainty factors of overall uncertainty by mean potentiality approach:

$$
\frac{1}{3} \sum_{i=1}^{3} C F_{T}\left(h_{i} / e_{1}, e_{2}, e_{3}\right)=0.4557 \text {. }
$$

By the definition, we calculate that the measure of performances by new approach and the mean potentiality approach are both $\Upsilon=3.8667$.

Briefly, the comparison results of above approaches are shown in the following table. 
Table 12: The comparison results of the substantial uncertainty factors of overall uncertainty

\begin{tabular}{cccc}
\hline Decision-making method & Result ranking & $C F_{U}$ & $\Upsilon$ \\
\hline Mean potentiality approach & $h_{2} \succ h_{3} \succ h_{1}$ & 0.4557 & 3.8667 \\
\hline New approach & $h_{2} \succ h_{3} \succ h_{1}$ & 0.3168 & 3.8667 \\
\hline
\end{tabular}

Analysis the results of above table, the value of the substantial uncertainty factors of overall uncertainty lower from the initial average 0.4557 to the combined 0.3168 .

Consider Example 3.2.2. We have

$$
\begin{gathered}
C F_{T}\left(h_{1} / e_{1}, e_{2}, e_{3}, e_{4}, e_{5}\right)=0.3909 \\
C F_{T}\left(h_{2} / e_{1}, e_{2}, e_{3}, e_{4}, e_{5}\right)=0.6669 \\
C F_{T}\left(h_{3} / e_{1}, e_{2}, e_{3}, e_{4}, e_{5}\right)=0.6734 \\
C F_{T}^{c}\left(h_{1} / e_{1}, e_{2}, e_{3}\right)=0.6091 \\
C F_{T}^{c}\left(h_{2} / e_{1}, e_{2}, e_{3}\right)=0.3331 \\
C F_{T}^{c}\left(h_{3} / e_{1}, e_{2}, e_{3}\right)=0.3264
\end{gathered}
$$

Then

$$
\frac{1}{3} \sum_{i=1}^{3} C F_{U}\left(h_{i} / e_{1}, e_{2}, e_{3}\right)=0.1542 .
$$

Calculate the substantial uncertainty factors of overall uncertainty by mean potentiality approach:

$$
\frac{1}{3} \sum_{i=1}^{3} C F_{T}\left(h_{i} / e_{1}, e_{2}, e_{3}\right)=0.5770 .
$$

By the definition, we can calculate that the measure of performances by new approach and the mean potentiality approach are both $\Upsilon=3.6954$.

Briefly, the comparison results of above approaches are shown in the following table.

Table 13: The comparison results of the substantial uncertainty factors of overall uncertainty

\begin{tabular}{cccc}
\hline Decision-making method & Result ranking & $C F_{U}$ & $\Upsilon$ \\
\hline Mean potentiality approach & $h_{3} \succ h_{2} \succ h_{1}$ & 0.5770 & 3.6954 \\
\hline New approach & $h_{3} \succ h_{2} \succ h_{1}$ & 0.1542 & 3.6954
\end{tabular}

Analysis the results of above table, the value of the substantial uncertainty factors of overall uncertainty lower from the initial average 0.5770 to the combined 0.1542 .
Consider Example 3.2.3. We have

$$
\begin{aligned}
& C F_{T}\left(h_{1} / e_{1}, e_{2}, e_{3}\right)=0.4841, \\
& C F_{T}\left(h_{2} / e_{1}, e_{2}, e_{3}\right)=0.7913, \\
& C F_{T}\left(h_{3} / e_{1}, e_{2}, e_{3}\right)=0.7270 ; \\
& C F_{T}^{c}\left(h_{1} / e_{1}, e_{2}, e_{3}\right)=0.5159, \\
& C F_{T}^{c}\left(h_{2} / e_{1}, e_{2}, e_{3}\right)=0.2087, \\
& C F_{T}^{c}\left(h_{3} / e_{1}, e_{2}, e_{3}\right)=0.2730 .
\end{aligned}
$$

Then

$$
\frac{1}{3} \sum_{i=1}^{3} C F_{U}\left(h_{i} / e_{1}, e_{2}, e_{3}\right)=0.3349 .
$$

Calculate the substantial uncertainty factors of overall uncertainty by mean potentiality approach:

$$
\frac{1}{3} \sum_{i=1}^{3} C F_{T}\left(h_{i} / e_{1}, e_{2}, e_{3}\right)=0.6674 .
$$

By the definition, we can calculate that the measure of performances by new approach and the mean potentiality approach are both $\Upsilon=4.1366$.

Briefly, the comparison results of above approaches are shown in the following table.

Table 14: The comparison results of the substantial uncertainty factors of overall uncertainty

\begin{tabular}{cccc}
\hline Decision-making method & Result ranking & $C F_{U}$ & $\Upsilon$ \\
\hline Mean potentiality approach & $h_{2}=h_{3} \succ h_{1}$ & 0.6674 & 4.1366 \\
\hline New approach & $h_{2} \succ h_{3} \succ h_{1}$ & 0.3349 & 4.1366
\end{tabular}

Analysis the results of above table, the value of the substantial uncertainty factors of overall uncertainty lower from the initial average 0.6674 to the combined 0.3349 .

The three examples above illustrate that new approach can significantly reduce the perception of uncertainty. Moreover, the measure of performances reflect the effectiveness of the new method. 


\section{An application for medical diagnosis problems}

One of the toughest challenges in medical diagnosis is handling uncertainty. Doctors always detect clinical manifestations by the comparison with predefined classes to find the most similar disease. Only one comprehensive result can be gotten from existing methods for medical diagnosis, which cannot provide the certainty or uncertainty of the result. Therefore, we do not know how to deal with the unknown factors in the process of medical diagnosis. In the section, we apply new approach to solve the medical diagnosis problem.

Now we consider the medical diagnosis problem cited from Examples 6.2 which is investigated by Basu et al. ${ }^{2}$.

Suppose that the universe $U$ contains four diseases, given by

$U=\{$ acute dental abscess, migraine, acute sinusitis, peritonsillar abscess $\}$

$=\left\{d_{1}, d_{2}, d_{3}, d_{4}\right\}$ (say).

And the set of parameters $E$ is given by

$E=$ fever, runningnose, weakness, orofacial pain, nausea vomiting, swelling, trismus, history, physical examination, laboratory investigation

$=\left\{e_{1}, e_{2}, e_{3}, e_{4}, e_{5}, e_{6}, e_{7}, s_{1}, s_{2}, s_{3}\right\}$ (say).

Let $A, B$ be two subsets of $E$ given by $A=$ $\left\{e_{1}, e_{2}, e_{3}, e_{4}, e_{5}, e_{6}, e_{7}\right\}$ and $B=\left\{s_{1}, s_{2}, s_{3}\right\}$. Suppose that $(F, A)$ and $(G, B)$ are two fuzzy soft sets describing "symptoms of the diseases" and "decision making tools of the diseases ", respectively.

We believe the authors of the literature ${ }^{2}$ have investigated some data and processed the data in Examples 6.2. And they get the tabular representation of $(F, A)$ and $(G, B)$, which are given in Tables 12 and 13 , respectively. In view of the rationality of the data, and in order to make better contrast with new approach, we quote the the tabular representation in Table 12 and 13.

Now suppose that a patient who is suffering from a disease has three symptoms as follows: fever, running nose, oro-facial pain, which is denoted by $P=e_{1}, e_{2}, e_{4}$. The key problem is how a doctor reaches to the most suitable diagnosis according to the symptoms, history, physical examina- tion and laboratory investigation of the patient. To solve this problem, we consider " $(F, A) \wedge(G, B)$ ", given by Table 14. There are four diseases $d_{1}, d_{2}, d_{3}, d_{4}$, and nine pairs of parameters $a_{1}=$ $\left(e_{1}, s_{1}\right), a_{2}=\left(e_{1}, s_{2}\right), a_{3}=\left(e_{1}, s_{3}\right), a_{4}=\left(e_{2}, s_{1}\right), a_{5}=$ $\left(e_{2}, s_{2}\right), a_{6}=\left(e_{2}, s_{3}\right), a_{7}=\left(e_{4}, s_{1}\right), a_{8}=\left(e_{4}, s_{2}\right), a_{9}=$ $\left(e_{4}, s_{3}\right)$, which is a pair of one symptom and one decision making tool, respectively.

Table 15: Tabular representation of the fuzzy soft set $(F, A)$

\begin{tabular}{cccccccc}
\hline & $e_{1}$ & $e_{2}$ & $e_{3}$ & $e_{4}$ & $e_{5}$ & $e_{6}$ & $e_{7}$ \\
\hline$d_{1}$ & 0.6 & 0 & 0.6 & 0.9 & 0 & 0.7 & 0.8 \\
$d_{2}$ & 0.2 & 0 & 0.1 & 0.9 & 0.8 & 0 & 0 \\
$d_{3}$ & 0.3 & 0.7 & 0.3 & 0.8 & 0.3 & 0.4 & 0 \\
$d_{4}$ & 0.4 & 0 & 0.2 & 0.7 & 0.1 & 0.6 & 0.5 \\
\hline
\end{tabular}

Table 16: Tabular representation of the fuzzy soft set $(G, B)$

\begin{tabular}{cccc}
\hline & $s_{1}$ & $s_{2}$ & $s_{3}$ \\
\hline$d_{1}$ & 0.6 & 0.8 & 0.4 \\
$d_{2}$ & 0.8 & 0.3 & 0.6 \\
$d_{3}$ & 0.8 & 0.4 & 0.7 \\
$d_{4}$ & 0.6 & 0.8 & 0.3 \\
\hline
\end{tabular}

Next we will apply new approach to detect which disease is most suited with the symptoms and these investigative procedures. Then, in the making decision, we consider that the four diseases construct a set of different assumptions, denoted by $D=\left\{d_{1}, d_{2}, d_{3}, d_{4}\right\}$. and the nine pairs of parameters as a set of evidences, which contains a diagnosis parameter system, denoted by $P=$ $\left\{a_{1}, a_{2}, a_{3}, a_{4}, a_{5}, a_{6}, a_{7}, a_{8}, a_{9}\right\}$.

Step 1. Construct the fuzzy soft matrix induced by " $(F, A) \wedge(G, B)$ ", which completely presents the degree that a patient is suffering from a disease $d_{i}$ with one symptom and one decision making tool $a_{j}$ as follows:

$$
G=\left(g_{i j}\right)_{4 \times 9}=\left(\begin{array}{ccccccccc}
0.6 & 0.6 & 0.4 & 0 & 0 & 0 & 0.6 & 0.8 & 0.4 \\
0.2 & 0.2 & 0.2 & 0 & 0 & 0 & 0.8 & 0.3 & 0.6 \\
0.3 & 0.3 & 0.3 & 0.7 & 0.4 & 0.7 & 0.8 & 0.4 & 0.7 \\
0.4 & 0.4 & 0.3 & 0 & 0 & 0 & 0.6 & 0.7 & 0.3
\end{array}\right) .
$$

Step 2. Construct the MYCIN certainty factor matrix as follows:

$$
\begin{aligned}
C F & =\left(s_{i j}\right)_{4 \times 9} \\
& =\left(\begin{array}{cccccccccc}
0.2 & 0.2 & -0.2 & -1 & -1 & -1 & 0.2 & 0.6 & -0.2 \\
-0.6 & -0.6 & -0.6 & -1 & -1 & -1 & 0.6 & -0.4 & 0.2 \\
-0.4 & -0.4 & -0.4 & 0.4 & -0.2 & 0.4 & 0.6 & -0.2 & 0.4 \\
-0.2 & -0.2 & -0.4 & -1 & -1 & -1 & 0.2 & 0.4 & -0.4
\end{array}\right)
\end{aligned}
$$


Table 17: Tabular representation of the fuzzy soft set $(F, A) \wedge(G, B)$

\begin{tabular}{cccccccccc}
\hline & $\left(e_{1}, s_{1}\right)$ & $\left(e_{1}, s_{2}\right)$ & $\left(e_{1}, s_{3}\right)$ & $\left(e_{2}, s_{1}\right)$ & $\left(e_{2}, s_{2}\right)$ & $\left(e_{2}, s_{3}\right)$ & $\left(e_{4}, s_{1}\right)$ & $\left(e_{4}, s_{2}\right)$ & $\left(e_{4}, s_{3}\right)$ \\
\hline$d_{1}$ & 0.6 & 0.6 & 0.4 & 0 & 0 & 0 & 0.6 & 0.8 & 0.4 \\
$d_{2}$ & 0.2 & 0.2 & 0.2 & 0 & 0 & 0 & 0.8 & 0.3 & 0.6 \\
$d_{3}$ & 0.3 & 0.3 & 0.3 & 0.7 & 0.4 & 0.7 & 0.8 & 0.4 & 0.7 \\
$d_{4}$ & 0.4 & 0.4 & 0.3 & 0 & 0 & 0 & 0.6 & 0.7 & 0.3 \\
\hline
\end{tabular}

Step 3. Since $a_{j}$ is specially more matching the mean of the parameter set than other parameters, $a_{j}$ contains the satisfying information for decision making and the uncertain degree of $a_{j}$ is low. Now, we consider the mean $\widetilde{s_{i}}$ of the parameter set with respect to $s_{i}$, calculated by $\widetilde{s_{i}}=\frac{1}{9} \sum_{j=1}^{9} s_{i j}$ as follows:

$$
\begin{gathered}
\widetilde{s_{1}}=-0.2444, \quad \widetilde{s_{2}}=-0.4889, \\
\widetilde{s_{3}}=0.0222, \quad \widetilde{s_{4}}=-0.4000 .
\end{gathered}
$$

Step 4. To obtain the gray mean relational degree, we need to calculate the difference information between $s_{i j}$ and $\widetilde{s_{i}}$ and construct the difference matrix as follows:

$$
\triangle S=\left(\begin{array}{ccccccccc}
0.4444 & 0.4444 & 0.0444 & 0.7556 & 0.7556 & 0.7556 & 0.4444 & 0.8444 & 0.0444 \\
0.1111 & 0.1111 & 0.1111 & 0.5111 & 0.5111 & 0.5111 & 1.0889 & 0.0889 & 0.6889 \\
0.4222 & 0.4222 & 0.4222 & 0.3778 & 0.2222 & 0.3778 & 0.5778 & 0.2222 & 0.3778 \\
0.2000 & 0.2000 & 0 & 0.6000 & 0.6000 & 0.6000 & 0.6000 & 0.8000 & 0
\end{array}\right)
$$

Step 5. Based on the the difference matrix $\triangle S$, the gray mean relational degree between $s_{i j}$ and $\widetilde{s_{i}}$ is calculated as follows:

$\left(r_{i j}\right)_{4 \times 9}=\left(\begin{array}{ccccccccc}0.5000 & 0.5000 & 0.8261 & 0.6667 & 0.5294 & 0.6667 & 1.0000 & 0.4035 & 0.8857 \\ 1.0000 & 1.0000 & 0.6552 & 0.8500 & 0.6750 & 0.8500 & 0.6054 & 1.0000 & 0.3333 \\ 0.5172 & 0.5172 & 0.3333 & 1.0000 & 1.0000 & 1.0000 & 0.8812 & 0.7931 & 0.4769 \\ 0.7895 & 0.7895 & 1.0000 & 0.7727 & 0.6136 & 0.7727 & 0.8641 & 0.4182 & 1.0000\end{array}\right)$

Step 6. Calculate the uncertain degree of each parameter $a_{j}$ as follows:

$$
\begin{array}{cc}
\operatorname{DOI}\left(a_{1}\right)=0.3658, & \operatorname{DOI}\left(a_{2}\right)=0.3658, \\
\operatorname{DOI}\left(a_{3}\right)=0.3727, & \operatorname{DOI}\left(a_{4}\right)=0.4156, \\
\operatorname{DOI}\left(a_{5}\right)=0.3634, & \operatorname{DOI}\left(a_{6}\right)=0.4156, \\
\operatorname{DOI}\left(a_{7}\right)=0.4250, & \operatorname{DOI}\left(a_{8}\right)=0.3506, \\
\operatorname{DOI}\left(a_{9}\right)=0.3643 .
\end{array}
$$

Step 7. Calculate the certain degree of each parameter $a_{j}$ as follows:

$$
C F\left(a_{1}\right)=0.6342, \quad C F\left(a_{2}\right)=0.6342,
$$

$$
\begin{array}{cl}
C F\left(a_{3}\right)=0.6273, & C F\left(a_{4}\right)=0.5844, \\
C F\left(a_{5}\right)=0.6366, & C F\left(a_{6}\right)=0.5844, \\
C F\left(a_{7}\right)=0.5750, & C F\left(a_{8}\right)=0.6494, \\
C F\left(a_{9}\right)=0.6357 .
\end{array}
$$

Step 8. Construct the essence uncertainty factor matrix as follows:

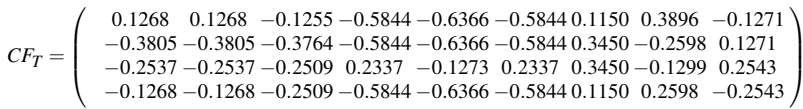

Step 9. Calculate respectively the confidence degree of each assumption $d_{i}$ by combining these evidences as follows:

$$
\begin{aligned}
& C F_{T}\left(h_{1} / e_{1}, e_{2}, e_{3}\right)=-0.9158, \\
& C F_{T}\left(h_{2} / e_{1}, e_{2}, e_{3}\right)=-0.9957, \\
& C F_{T}\left(h_{3} / e_{1}, e_{2}, e_{3}\right)=0.0623, \\
& C F_{T}\left(h_{4} / e_{1}, e_{2}, e_{3}\right)=-0.9861 .
\end{aligned}
$$

Then the final rang order is $d_{3} \succ d_{1} \succ d_{4} \succ d_{2}$. According to the maximum confidence degree principle, the patient is suffering from acute sinusitis $d_{3}$, which is the same choice decision based on the mean potentiality approach of Example 6.2 investigated by Basu et al. ${ }^{2}$. Thus, we can push out this consequence is reasonable and effective.

Next, we consider the overall uncertainty of this medical diagnosis problem.

We have

$$
\begin{gathered}
C F_{T}\left(h_{1} / e_{1}, e_{2}, e_{3}\right)=-0.9158, \\
C F_{T}\left(h_{2} / e_{1}, e_{2}, e_{3}\right)=-0.9957, \\
C F_{T}\left(h_{3} / e_{1}, e_{2}, e_{3}\right)=0.0623 \\
C F_{T}\left(h_{4} / e_{1}, e_{2}, e_{3}\right)=-0.9861 \\
C F_{T}^{c}\left(h_{1} / e_{1}, e_{2}, e_{3}\right)=0.0842 \\
C F_{T}^{c}\left(h_{2} / e_{1}, e_{2}, e_{3}\right)=0.0043
\end{gathered}
$$




$$
\begin{aligned}
& C F_{T}^{c}\left(h_{3} / e_{1}, e_{2}, e_{3}\right)=0.9377 . \\
& C F_{T}^{c}\left(h_{4} / e_{1}, e_{2}, e_{3}\right)=0.0139,
\end{aligned}
$$

Then

$$
\frac{1}{4} \sum_{i=1}^{4} C F_{U}\left(h_{i} / e_{1}, e_{2}, e_{3}\right)=0.4799
$$

Calculate the substantial uncertainty factors of overall uncertainty by mean potentiality approach:

$$
\frac{1}{4} \sum_{i=1}^{4} C F_{T}\left(h_{i} / e_{1}, e_{2}, e_{3}\right)=0.7399 .
$$

By the definition, we calculate that the measure of performances by new approach and the mean potentiality approach are both $\Upsilon=4.7163$.

Briefly, the comparison results of above approaches are shown in the following table.

Table 18: The comparison results of the substantial uncertainty factors of overall uncertainty

\begin{tabular}{cccc}
\hline Decision-making method & Result ranking & $C F_{U}$ & $\Upsilon$ \\
\hline Mean potentiality approach & $h_{3} \succ h_{1} \succ h_{4} \succ h_{2}$ & 0.7399 & 4.7163 \\
\hline New approach & $h_{3} \succ h_{1} \succ h_{4} \succ h_{2}$ & 0.4799 & 4.7163 \\
\hline
\end{tabular}

Analysis the results of above tables, the value of the substantial uncertainty factors of overall uncertainty lower from the initial average 0.7399 to the combined 0.4799 , which illustrate that new approach can significantly reduce the perception of uncertainty. Moreover, the measure of performances reflect the effectiveness of the new method.

\section{Conclusions}

In this paper, we have introduced a new approach to fuzzy soft sets in decision making by combining grey relational analysis with MYCIN certainty factor and given a practical application to medical diagnosis problems. If we first apply grey relational analysis to construct suitable essence uncertainty factor for each assumption and then use MYCIN certainty factor combination rule to compose these information, the confidence degree of the whole uncertainty is declined. In a sense, this approach can help reducing uncertainty caused by people's subjective cognition so as to raise the choice decision level. Moreover, compared with the mean potentiality approach, this approach allows us to avoid this problem of selecting the suitable level soft set and are more feasible and practical for dealing with the real-life application under uncertainty. Besides, this approach sets up a decision making model and thus broadens the application field of the grey system theory. Our future work will concentrate on its application to interval-valued intuitionistic fuzzy soft sets in decision making.

\section{Acknowledgements}

The authors would like to thank the editors and the anonymous reviewers for their valuable comments and suggestions which have helped immensely in improving the quality of this paper. This work is supported by the National Natural Science Foundation of China (11461005, 11201490, 11401052), the Natural Science Foundation of Guangxi (2014GXNSFAA118001), Guangxi University Science and Technology Research Project (KY2015YB075, KY2015YB081,KY2015YB266), Quantitative Economics Key Laboratory Program of Guangxi University of Finance and Economics (2014SYS11), the Science Research Project 2014 of the China-ASEAN Study Center (Guangxi Science Experiment Center) of Guangxi University for Nationalities (KT201427), the Research Project 2014 of Teacher Education (2014JS013) and China Postdoctoral Science Foundation (2013M542558).

\section{References}

1. H.Aktas, N.Cağman, "Soft sets and soft groups," Information Sciences, 177, 2726-2735 (2007).

2. T.M.Basu, N.K.Mahapatrab, S.K.Mondal, "A balanced solution of a fuzzy soft set based decision making problem in medical science," Applied Soft Computing, 12, 3260-3275 (2012).

3. N.Ca ğman, S.Engino ğlu, "Soft matrix theory and its decision making," Computers and Mathematics with Applications, 59, 3308-3314 (2010).

4. N.Ca ğman, S.Engino ğlu, "Soft set theory and uni-int decision making," European Journal of Operational Research, 207, 848-855 (2010).

5. D.Chen, E.C.C.Tsang, D.Yeung, X.Wang, "The parameterization reduction of soft sets and its applications," Computers and Mathematics with Applications, 49, 757-763 (2005). 
6. J.Deng, "The introduction of grey system," The Journal of Grey System, 1, 1-24 (1989).

7. D.Heckerman, "Probablistic interpretations for MYCIN's certainty factors," Morgan Kaufmann Publishers, San Francisco, 1990.

8. F.Feng, Y.Li, V.Leoreanu-Fotea, "Application of level soft sets in decision making based on interval-valued fuzzy soft sets," Computers and Mathematics with Applications, 60, 1756-1767 (2010).

9. F.Feng, Y.B.Jun, X.Liu, L.Li, “An adjustable approach to fuzzy soft set based decision making," Journal of Computational and Applied Mathematics, 234, 10-20 (2010).

10. F.Feng, Y.B.Jun, X.Zhao, "Soft semirings," Computers and Mathematics with Applications, 56, 26212628 (2008).

11. Z.Gong, L.Li, F.Zhou, T.Yao, "Goal programming approaches to obtain the priority vectors from the intuitionistic fuzzy preference relations," Computers and industrial Engineering, 57, 1187-1193 (2009).

12. Z.Gong, L.Li, J.Cao, F.Zhou, "On additive consistent properties of the intuitionistic fuzzy preference relation," International Journal of Information Technology and Decision Making, 9, 1009-1025 (2010).

13. Z.Gong, L.Li, J.Forrest, Y.Zhao, "The optimal priority models of the intuitionistic fuzzy preference relation and their application in selecting industries with higher meteorological sensitivity," Expert Systems with Applications, 38, 4394-4402 (2011).

14. Z.Gong, J.Forrest, Y.Yang, "The optimal group consensus models for 2-tuple linguistic preference relations," Knowledge-Based Systems, 37, 427-437 (2013).

15. T.Herawan, M.M.Deris, "A soft set approach for association rules mining," Knowledge-Based Systems, 24, 186-195 (2011).

16. Y.B.Jun, "Soft BCK/BCI-algebras," Computers and Mathematics with Applications, 56, 1408-1413 (2008).

17. Y.B.Jun, K.J.Lee, C.H.Park, "Fuzzy soft set theory applied to BCK/BCI-algebras," Computers and Mathematics with Applications, 59, 3180-3192 (2010).

18. Y.Jiang, Y.Tang, Q.Chen, "An adjustable approach to intuitionistic fuzzy soft sets based decision making," Applied Mathematical Modelling, 35, 824-836 (2011).

19. Y.Jiang, Y.Tang, Q.Chen, J.Wang, S.Tang, "Extending soft sets with description logics," Computers and Mathematics with Applications, 59(2010), $2087-$ 2096.

20. Z.Kong, L.Gao, L.Wang, "Comment on a fuzzy soft set theoretic approach to decisionmaking problems," Journal of Computational and Applied Mathematics, 223, 540-542 (2009).

21. S.J.Kalayathankal, G.S.Singh, "A fuzzy soft flood alarm model," Mathematics and Computers in Simulation, 80, 887-893 (2010).

22. C.Kung, K.Wen, "Applying grey relational analysis and grey decision-making to evaluate the relationship between company attributes and its financial performance-A case study of venture capital enterprises in Taiwan," Decision Support Systems, 43, 842852 (2007).

23. Z.Kong, L.Wang, Z.Wu, "Application of fuzzy soft set in decision making problems based on grey theory," Journal of Computational and Applied Mathematics, 236, 1521-1530 (2011).

24. S.Liu, Y.Dang, Z.Fang, "Grey systems theory and its applications," Science Press, Beijing, 2004.

25. H.Liu, J.Liu, "Expert System C++ program design based on MYCIN inexect inference," Computer Engineering and Design, 22, 47-55 (2001).

26. P.Li, S.Liu, Z.Fang, "Interval-valued intuitionistic fuzzy numbers decision-making methed based on grey incidence analysis and MYCIN certainty factor," Control and Decision, 27, 1009-1014 (2012).

27. Z.Li, T.Xie, "Roughness of fuzzy soft sets and related results," International Journal of Computational Intelligence Systems, 8, 278-296 (2015).

28. Z.Li, T.Xie, "The relationship among soft sets, soft rough sets and topologies," Soft Computing, 18, 717728 (2014).

29. Z.Li, G.Wen, Y.Han, "Decision making based on intuitionistic fuzzy soft sets and its algorithm," Journal of Computational Analysis and Applications, 17, 620631 (2014)

30. Z.Li, N.Xie, G.Wen, "Soft coverings and their parameter reductions," Applied Soft Computing, 31, 48-60 (2015).

31. D.Molodtsov, "Soft set theory-First result," Computers and Mathematics with Applications, 37, 19-31 (1999).

32. P.K.Maji, R.Biswas, A.R.Roy, "Fuzzy soft sets," The Journal of Fuzzy Mathematics, 9, 589-602 (2001).

33. P.K.Maji, R.Biswas, A.R.Roy, "Intuitionistic fuzzy soft sets," Journal of Fuzzy Mathematics, 9, 677-692 (2001).

34. P.K.Maji, R.Biswas, A.R.Roy, "Soft set theory," Computers and Mathematics with Applications, 45, 555562 (2003).

35. P.K.Maji, A.R.Roy, "An application of soft sets in a decision making problem," Computers and Mathematics with Applications, 44, 1077-1083 (2002).

36. X.Ma, N.Sulaiman, H.Qin, T.Herawan, J.M.Zain, “A new efficient normal parameter reduction algorithm of soft sets," Computers and Mathematics with Applications, 62, 588-598 (2011).

37. Z.Pawlak, "Rough sets," International Journal of Computing and Information Sciences, 11, 341-356 (1982). 
38. A.R.Roy, P.K.Maji, "A fuzzy soft set theoretic approach to decision making problems," Journal of Computational and Applied Mathematics, 203, 412418 (2007).

39. E.H.Shortliffe, B.G.Buchanan, "A model of inexact reasoning in medicine," Mathematical Biosciences, 23, 351-379 (1975).

40. E.H.Shortliffe, "Computer based medical consultations: MYCIN," Elsevier, New York, 1976.

41. L.Sui, B.Luo, D.Shao, "D-S based investment decision model and its application," Systerms Engineering, 20, 71-76 (2002).

42. G.Wei, "Gray relational analysis method for intuitionistic fuzzy multiple attribute decision making," Expert Systems with Applications, 38, 11671-11677 (2011).

43. Z.Xiao, K.Gong, Y.Zou, "A combined forecasting approach based on fuzzy soft sets," Journal of Computational and Applied Mathematics, 228, 326-333 (2009).

44. W.Xu, J.Ma, S.Wang, G.Hao, "Vague soft sets and their properties," Computers and Mathematics with Applications, 59, 787-794 (2010).

45. X.Yang, T.Lin, J.Yang, Y.Li, D.Yu, "Combination of interval-valued fuzzy set and soft set," Computers and Mathematics with Applications, 58, 521-527 (2009).

46. L.A.Zadeh, "Fuzzy sets," Information and Control, 8, 338-353 (1965).

47. X.Zhang, J.Dai, Y.Yu, "On the union and intersection operations of rough sets based on various approximation spaces," Information Sciences, 292, 214-229 (2015)

48. X.Zhang, H.Zhou, X.Mao, "IMTL(MV)-filters and fuzzy IMTL(MV)-filters of residuated lattices," Journal of Intelligent and Fuzzy Systems, 26, 589-596 (2014).

49. W.Zhang, Y.Liang, P.Xu, "Uncertainty Reasoning Based on Inclusion Degree," Tsinghua University Press, Beijing, 2007.

50. J.Zhang, D.Wu, D.L.Olson, "The method of grey related analysis to multiple attribute decision making problems with interval numbers," Mathematical and Computer Modelling, 42, 991-998 (2005).

51. Y.Zou, Z.Xiao, "Data analysis approaches of soft sets under incomplete information," Knowledge-Based Systems, 21, 941-945 (2008). 